\title{
38 Floor Apartment Design with Special Moment Frame System (SMF), With Performance Based Design Using SNI 1726: 2019 AND SNI 1729: 2015
}

\author{
Achmad Ubaidillah ${ }^{1}$, Tony Hartono Bagio ${ }^{2}$, Julistyana Tistogondo ${ }^{3}$ \\ Departement of Civil Engineering, Faculty of Engineering \\ Narotama University, Surabaya, Indonesia 1,2,3 \\ ubaiduniversal@gmail.com¹ ${ }^{1}$, tony@narotama.ac.id ${ }^{2}$, \\ julistyana.tistogondo@narotama.ac.id ${ }^{3}$
}

\begin{abstract}
Apartments are an option for vertical housing. Currently, with the rapid development of technology, a person will tend to choose housing that can support their mobility. So it is planned that an apartment building structure is planned using a Special Moment Frame System using Performance Based

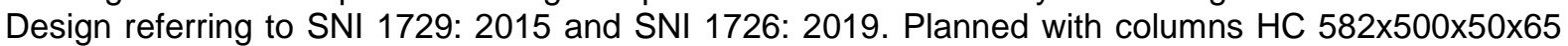

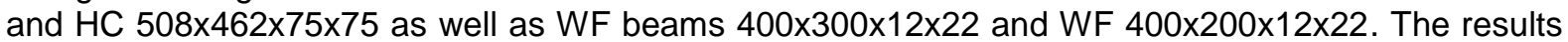
show that the columns and beams are strong but wasteful.
\end{abstract}

Keywords: Apartment, Special Momen Frame, Performance Based Design, SNI 1729, SNI 1726

\section{INTRODUCTION}

\subsection{Overview}

The very high population growth creates new problems in urban planning. This is based on the large number of urbanization of people from rural areas to urban areas, factors such as education, trade and technology support become the reason someone thinks of living in a big city. Adequate and appropriate occupancy is needed by paying attention to many aspects based on the local population. In today's digital era, residential needs are still a priority in life. Knowing this, the local government is certainly looking for a solution in future city planning considering the limited amount of land available. One solution that has been implemented is to limit residential construction horizontally, and to promote vertical housing development.

The apartment is one of the options for vertical housing. At present, with the rapid development of technology, someone will tend to choose a residence that can support their mobility, someone will tend to choose a residence that is located in the middle of the city and close to the economic center because their residence will be considered to have great value compared to housing in the suburbs. Apartments will be a solution to these problems considering that the land required for apartment construction is not as large as public housing. And now there are many apartments that offer convenience with locations that are in the same area as office buildings and shopping centers. Because of this, apartments are suitable as an alternative to people's needs for housing.

From some of the problems above, planning a steel building structure for an earthquakeresistant apartment plan with a Special Moment Frame System (SMF), engineering using Performance Based Design and SNI 1729: 2015 and SNI 1726: 2019 standards.

\section{LITERATURE REVIEW}

Structural performance is the level of performance of a structure against a planned earthquake. The level of structural performance can be determined by looking at the level of damage to the structure when it is hit by a planned earthquake with a certain return period. In performance-based design, the building structure is designed by considering the condition of the building during an earthquake and the safety of the building occupants.

There have been many developing methods of designing buildings, the most commonly used method is based on the ultimate force. The performance-based method is actually well known, but in Indonesia this method is relatively new and there is not much literacy that discusses this method when compared to the ultimate force-based method. 


\section{METHODOLOGY}

\subsection{Flow Chart}

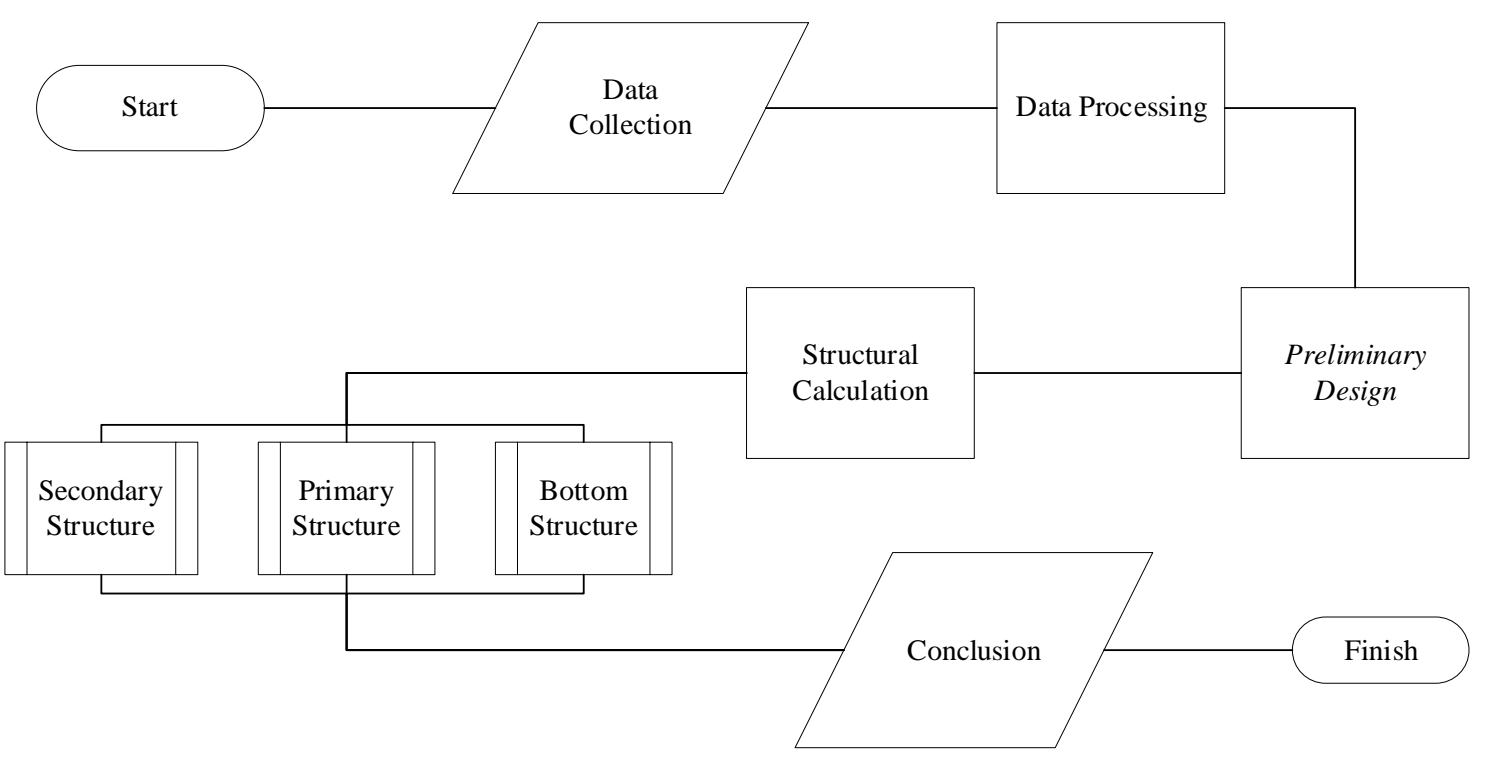

Picture 1. Flow Chart

\section{RESULT AND DISCUSSION}

\subsection{Seismic Classification}

4.1.1. Determining the Classification of Soil Sites

$$
\begin{aligned}
\bar{N} & =\sum_{i=1}^{n} d_{i} / \sum_{i=1}^{n} \frac{d_{i}}{N_{i}} \\
& =\frac{55}{3.11} \\
& =17.68
\end{aligned}
$$

Based on the soil classification in SNI 1726: 2019 Table 5, the value of $\bar{N}$ $=17.68$ is categorized as a class of SD sites (medium soil).

\subsubsection{Determining Risk Categories}

The risk category is stated based on the function of the building itself, as the building function is an apartment, so based on SNI 1726: 2019 the risk category is stated in category II.

\subsubsection{Determining Seismic Design Categories}

The values of Ss (short period earthquake acceleration spectral response parameter 0.2 seconds) and $S 1$ (earthquake acceleration spectral response parameter short period 1 second) were determined using the application "Design Spectra Indonesia" from puskim.pu.go.id then the following results were obtained. 
Table 1. Result Table "Design Spectra Indonesia"

\begin{tabular}{|c|c|}
\hline Parameter & Medium Soil D \\
\hline$S_{s}$ & 0.665 \\
\hline$S_{1}$ & 0.252 \\
\hline$S_{M S}$ & 0.844 \\
\hline$S_{M 1}$ & 0.477 \\
\hline$S_{D S}$ & 0.562 \\
\hline$S_{D 1}$ & 0.318 \\
\hline
\end{tabular}

Source : http://puskim.pu.go.id/

With SS and S1 data in the table above and the requirements in tables 8 and 9 of SNI 1726: 2019), the earthquake design category can be determined as follows;

a. Medium Soil

SDS $=0.562$

Seismic Design Category D for risk category II.

$\mathrm{SD} 1=0.318$

Seismic Design Category D for risk category II.

From the results of the data above based on SNI 1726: 2019, the seismic design category is categorized as $\mathrm{D}$ for buildings with risk category II.

\subsubsection{Determining the Priority Factors of the Earthquake}

The earthquake priority factor is stated based on the building risk category, the apartment building risk category is II, so the earthquake priority factor le is 1.0.

\subsubsection{Determining Site Coefficients}

The Fa and Fv site coefficients follow table 6 SNI 1726: 2019 and Ss, S1 from the calculation results of the "Indonesian Spectra Design" application, then interpolation is carried out as follows.

Table 2. Fa Site Coefficient

\begin{tabular}{|lll|}
\hline & $\boldsymbol{S}_{s}=\mathbf{0 , 5}$ & $\boldsymbol{S}_{s}=\mathbf{0 , 7 5}$ \\
\hline$S D$ & 1,4 & 1,2 \\
\hline
\end{tabular}

If $S_{s}=0.665$ then,

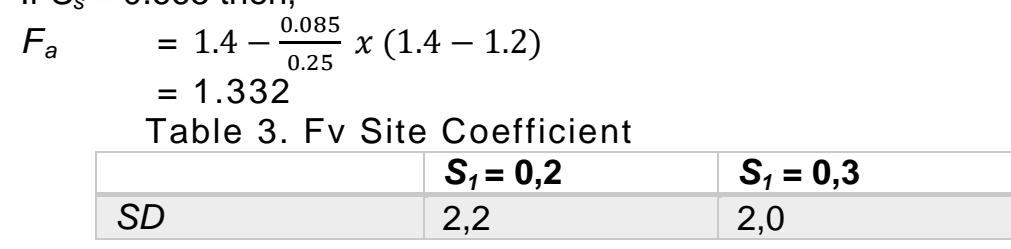

If $S_{1}=0.252$ then,

$F_{v} \quad=2.2-\frac{0.048}{0.1} x(2.2-2.0)$ 
4.2. Load Analysis

4.2.1. Dead Load

Table 4 : Total Dead Loads

\begin{tabular}{|c|c|}
\hline Story & Total Loads (kN) \\
\hline \multicolumn{2}{|c|}{ Total } \\
\hline Story 1 & 19178.49972 \\
\hline Story 2 & 8068.586782 \\
\hline Story 3 & 11030.29782 \\
\hline Story 4 & 9393.861826 \\
\hline Story 5-7 & 30689.44106 \\
\hline Story 8 & 12404.6693 \\
\hline Story 9 & 1633.362501 \\
\hline Story 10 & 3504.027778 \\
\hline Story 11 & 4514.337249 \\
\hline Story $12-20$ & 41772.48443 \\
\hline Story 21 & 3778.451789 \\
\hline Story $22-30$ & 41772.48443 \\
\hline Story 31 & 4174.833969 \\
\hline Story $32-38$ & 36664.54408 \\
\hline Roof & 3195.787281 \\
\hline TOTAL & 231775.67 \\
\hline
\end{tabular}

4.2.2. Live Load

Table 5 : Total Live Loads

\begin{tabular}{|c|c|}
\hline Story & $\begin{array}{l}\text { Total Loads } \\
\text { (kN) }\end{array}$ \\
\hline \multicolumn{2}{|c|}{ Total } \\
\hline Story 1 & 9997.9176 \\
\hline Story 2 & 3919.7136 \\
\hline Story 3 & 5607.492 \\
\hline Story 4 & 4693.4448 \\
\hline Story 5-7 & 15467.364 \\
\hline Story 8 & 6423.12 \\
\hline Story 9 & 607.3848 \\
\hline Story 10 & 1727.7744 \\
\hline Story 11 & 2303.2152 \\
\hline Story $12-20$ & 20728.9368 \\
\hline Story 21 & 1822.2072 \\
\hline Story $22-30$ & 20728.9368 \\
\hline Story 31 & 2040.2712 \\
\hline Story 32-38 & 16122.5064 \\
\hline Roof & 748.4832 \\
\hline TOTAL & 112938.768 \\
\hline $\begin{array}{l}\text { TOTAL * } 1 / 2 \\
\text { (reduction factor) }\end{array}$ & 56469.384 \\
\hline
\end{tabular}




\subsubsection{Seismic Load}

The effective seismic weight is taken from 1 total dead load +0.5 total live load.

Table 6 : Effective Seismic Weight

\begin{tabular}{|l|r|r|r|}
\hline Story & \multicolumn{1}{|c|}{$\begin{array}{c}\text { Dead Loads } \\
(\mathrm{kN})\end{array}$} & \multicolumn{1}{c|}{$\begin{array}{c}\text { Live Loads } \\
(\mathrm{kN})\end{array}$} & $\begin{array}{c}\text { Total Loads } \\
(\mathrm{kN})\end{array}$ \\
\hline Story 32-38 & 36664.54408 & 8061.2532 & 44725.7973 \\
\hline Story 31 & 4174.833969 & 1020.1356 & 5194.96957 \\
\hline Story 22-30 & 41772.48443 & 10364.4684 & 52136.9528 \\
\hline Story 21 & 3778.451789 & 911.1036 & 4689.55539 \\
\hline Story 12-20 & 41772.48443 & 10364.4684 & 52136.9528 \\
\hline Story 11 & 4514.337249 & 1151.6076 & 5665.94485 \\
\hline Story 10 & 3504.027778 & 863.8872 & 4367.91498 \\
\hline Story 09 & 1633.362501 & 303.6924 & 1937.0549 \\
\hline Story 08 & 12404.6693 & 3211.56 & 15616.2293 \\
\hline Story 05-7 & 30689.44106 & 7733.682 & 38423.1231 \\
\hline Story 04 & 9393.861826 & 2346.7224 & 11740.5842 \\
\hline Story 03 & 11030.29782 & 2803.746 & 13834.0438 \\
\hline Story 02 & 8068.586782 & 1959.8568 & 10028.4436 \\
\hline Story 01 & 19178.49972 & 4998.9588 & 24177.4585 \\
\hline Roof & 3195.787281 & 374.2416 & 3570.02888 \\
\hline TOTAL & 231775.67 & 56469.384 & 288245.054 \\
\hline
\end{tabular}

4.2.3.1. Slide the seismic base

$$
\begin{aligned}
\mathrm{C}_{\mathrm{s}} & =\mathrm{S}_{\mathrm{DS}} /\left(\mathrm{R} / \mathrm{I}_{\mathrm{e}}\right) \\
& =0.59 /(8 / 1) \\
& =0.07375 \\
\mathrm{~V} & =\mathrm{C}_{\mathrm{s}} \mathrm{W} \\
& =0.07375 \times 288245.054 \\
& =21258.07
\end{aligned}
$$

4.2.3.2. Distribution of seismic forces

$$
\begin{aligned}
& \mathrm{F}_{\mathrm{x}}=\mathrm{C}_{\mathrm{vx}} \mathrm{V} \\
& \text { and } \\
& \mathrm{C}_{\mathrm{vx}}=w_{x} h_{x}^{k} / \sum_{i=l}^{n} w_{i} h_{i}^{k}
\end{aligned}
$$

Table 7 : Total Seismic Loads

\begin{tabular}{|c|c|c|c|c|c|}
\hline Story & $\mathrm{H}(\mathrm{m})$ & $\mathrm{W}(\mathrm{kN})$ & W.hxk & Cvx & $\mathrm{Fx}$ \\
\hline Roof & 128.2 & 3570.029 & 58674281.5 & 0.046382341 & 198.4573 \\
\hline Story 38 & 124.8 & 6389.4 & 99515154.5 & 0.078667275 & 336.5957 \\
\hline Story 37 & 121.4 & 6389.4 & 94166715.9 & 0.074439305 & 318.5054 \\
\hline Story 36 & 118 & 6389.4 & 88966000.2 & 0.070328111 & 300.9147 \\
\hline Story 35 & 114.6 & 6389.4 & 83913007.4 & 0.066333692 & 283.8237 \\
\hline Story 34 & 111.2 & 6389.4 & 79007737.5 & 0.062456049 & 267.2323 \\
\hline Story 33 & 107.8 & 6389.4 & 74250190.6 & 0.058695183 & 251.1406 \\
\hline Story 32 & 104.4 & 6389.4 & 69640366.6 & 0.055051091 & 235.5485 \\
\hline
\end{tabular}


VOLUME 20 | NUMBER 2 | JANUARY 2021 https://ejournal.worldconference.id/index.php/neutron

E-ISSN: 2685-3272 | P-ISSN 1412-0860

\begin{tabular}{|c|c|c|c|c|c|}
\hline Story & $\mathrm{H}(\mathrm{m})$ & $\mathrm{W}(\mathrm{kN})$ & $\mathrm{W} . \mathrm{hxk}$ & $\mathrm{Cvx}$ & $\mathrm{Fx}$ \\
\hline Story 31 & 101 & 5194.97 & 52993884.6 & 0.041891956 & 179.2442 \\
\hline Story 30 & 97.6 & 5792.995 & 55182677.8 & 0.043622209 & 186.6475 \\
\hline Story 29 & 94.2 & 5792.995 & 51404950 & 0.040635895 & 173.8699 \\
\hline Story 28 & 90.8 & 5792.995 & 47761156.3 & 0.037755456 & 161.5452 \\
\hline Story 27 & 87.4 & 5792.995 & 44251296.6 & 0.034980892 & 149.6737 \\
\hline Story 26 & 84 & 5792.995 & 40875371 & 0.032312205 & 138.2551 \\
\hline Story 25 & 80.6 & 5792.995 & 37633379.4 & 0.029749393 & 127.2895 \\
\hline Story 24 & 77.2 & 5792.995 & 34525321.9 & 0.027292456 & 116.7769 \\
\hline Story 23 & 73.8 & 5792.995 & 31551198.4 & 0.024941395 & 106.7174 \\
\hline Story 22 & 70.4 & 5792.995 & 28711008.9 & 0.02269621 & 97.11086 \\
\hline Story 21 & 67 & 4689.555 & 21051414.1 & 0.016641258 & 71.20338 \\
\hline Story 20 & 63.6 & 5792.995 & 23432432.1 & 0.018523466 & 79.25683 \\
\hline Story 19 & 60.2 & 5792.995 & 20994044.7 & 0.016595907 & 71.00934 \\
\hline Story 18 & 56.8 & 5792.995 & 18689591.4 & 0.014774224 & 63.21486 \\
\hline Story 17 & 53.4 & 5792.995 & 16519072.1 & 0.013058417 & 55.87339 \\
\hline Story 16 & 50 & 5792.995 & 14482486.9 & 0.011448485 & 48.98493 \\
\hline Story 15 & 46.6 & 5792.995 & 12579835.7 & 0.009944429 & 42.54949 \\
\hline Story 14 & 43.2 & 5792.995 & 10811118.5 & 0.008546248 & 36.56705 \\
\hline Story 13 & 39.8 & 5792.995 & 9176335.42 & 0.007253943 & 31.03764 \\
\hline Story 12 & 36.4 & 5792.995 & 7675486.34 & 0.006067514 & 25.96123 \\
\hline Story 11 & 33 & 5665.945 & 6170213.94 & 0.004877588 & 20.86986 \\
\hline Story 10 & 30 & 4367.915 & 3931123.48 & 0.003107575 & 13.29646 \\
\hline Story 9 & 27 & 1937.055 & 1412113.02 & 0.001116283 & 4.776269 \\
\hline Story 8 & 24 & 15616.23 & 8994948.08 & 0.007110556 & 30.42412 \\
\hline Story 7 & 21 & 12807.71 & 5648199.09 & 0.004464932 & 19.10422 \\
\hline Story 6 & 18 & 12807.71 & 4149697.29 & 0.003280358 & 14.03575 \\
\hline Story 5 & 15 & 12807.71 & 2881734.23 & 0.002278027 & 9.747052 \\
\hline Story 4 & 12 & 11740.58 & 1690644.13 & 0.001336463 & 5.718361 \\
\hline Story 3 & 9 & 13834.04 & 1120557.55 & 0.000885807 & 3.790125 \\
\hline Story 2 & 6 & 10028.44 & 361023.969 & 0.000285391 & 1.221112 \\
\hline Story 1 & 3 & 24177.46 & 217597.127 & 0.000172012 & 0.735991 \\
\hline & & 288245.1 & 1265013368 & 1 & 4278.726 \\
\hline
\end{tabular}

4.3. Frame Analysis

4.3.1. Plan Profile

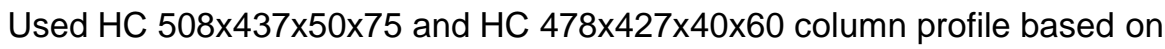

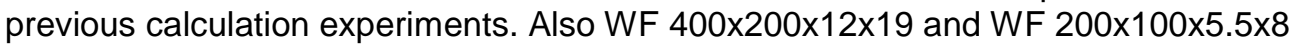
based on previous calculation experiments. 
Table 8 : Column and Beam Forces (from ETABS)

\begin{tabular}{|c|c|c|c|c|}
\hline Frame & Function & $\mathrm{Pu}(\mathrm{kN})$ & $\mathrm{Mu}(\mathrm{kN})$ & $\mathrm{Vu}(\mathrm{kN})$ \\
\hline HC 508x437x50x75 & Column & 13393.1 & 637.8 & 377.6 \\
\hline HC 478x427x40x60 & Column & 10092.5 & 747.4 & 328.46 \\
\hline WF $400 \times 200 \times 12 \times 19$ & Beam & 240.7 & 282.85 & 136 \\
\hline WF $200 \times 100 \times 5.5 \times 8$ & Beam & 0.14 & 35 & 26.68 \\
\hline
\end{tabular}

Table 9 : Column and Beam Capacity

\begin{tabular}{|c|c|c|c|c|}
\hline Frame & Function & $\mathrm{Pu}(\mathrm{kN})$ & $\mathrm{Mu}(\mathrm{kN})$ & $\mathrm{Vu}(\mathrm{kN})$ \\
\hline HC 508x437×50x75 & \begin{tabular}{|l} 
Column \\
\end{tabular} & 17744 & 3885.1 & 2397.1 \\
\hline HC 478x427x40x60 & Column & 13903.7 & 4320.3 & 1917.7 \\
\hline WF $400 \times 200 \times 12 \times 19$ & Beam & 2002 & 410 & 581.7 \\
\hline WF $200 \times 100 \times 5.5 \times 8$ & Beam & 142.7 & 44.67 & 135.5 \\
\hline
\end{tabular}

HC 508x437x50x75 Pu capacity / force

HC 478x427x40x60 Pu capacity / force

WF 400x200x12×19 Mu capacity / force

WF $200 \times 100 \times 5.5 \times 8$ Mu capacity / force

$$
\begin{aligned}
& =17744 / 13393.1 \\
& =1.32 \\
& =13903.7 / 10092.5 \\
& =1.37 \\
& =410 / 282.85 \\
& =1.44 \\
& =44.67 / 35 \\
& =1.27
\end{aligned}
$$

All beams and collums capacity / force rasio are OK.

\subsection{Pushover}

In pushover analysis, ETABS 17 is used and the following results are obtained. At the maximum displacement an assumption is used of $2 \%$ of the building height.

\section{- $\quad$ Non Linear Case "GRAVITY"}

Create a new load case of nonlinear static type. At this stage it is assumed that the scale factor for dead load is 1 and for live load is $1 / 2$.

- Non Linear Case "PUSHX"

Create a new load case of nonlinear static type. Use load type acceleration UX with a scale factor of -1 .

In the load application data input is as follows. The result of $2,564 \mathrm{~m}$ is assumed to be $2 \%$ of the building height. The 2431 joint label is obtained at the point closest to the center of the diaphragm on the top floor.

- Non Linear Case "PUSHY"

Similar to PUSH X, it creates a new load case of type nonlinear static. Use load type acceleration UY with a scale factor of -1 .

In the load application data input is as follows. The result of 2,564 $\mathrm{m}$ is assumed to be $2 \%$ of the building height. The 2431 joint label is obtained at the point closest to the center of the diaphragm on the top floor.

- Hinge Assign

For defining plastic joints that occur in each element as follows.

$\circ$ Column = P-M2-M3 
E-ISSN: 2685-3272 | P-ISSN 1412-0860

In the column defining the plastic hinge it is only used on the ground floor column only. In each assign plastic hinge, relative distance 1 and 0 are used.

$\circ$ Beam = $\mathrm{M} 3$

In each assign plastic hinge, relative distance 1 and 0 are used.

The next step is to run the ETABS 17 program on the nonlinear load case "GRAVITY", "PUSHX", "PUSHY" and get the following results.

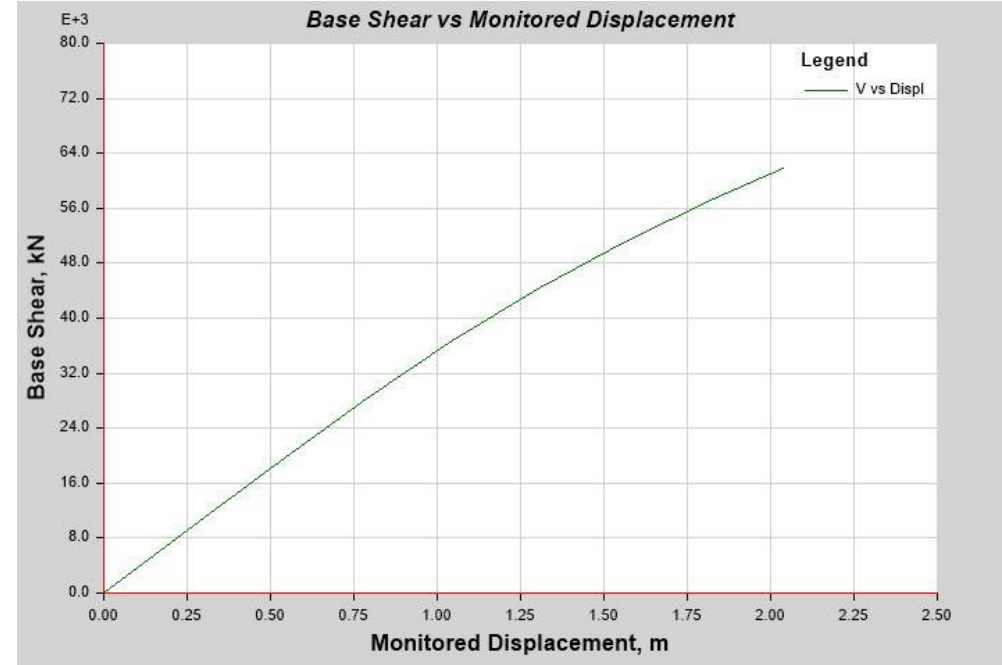

Picture 12. Graphic Displacement X direction

Table 10 : Result Table Displacement X

\begin{tabular}{|c|c|c|c|c|c|c|c|c|c|c|c|c|}
\hline \multicolumn{4}{|c|}{$\begin{array}{c}\text { TABLE: Base Shear vs Monitored } \\
\text { Displacement }\end{array}$} & \multirow[b]{2}{*}{$\begin{array}{l}\text { B- } \\
\text { C }\end{array}$} & \multirow[b]{2}{*}{$\begin{array}{l}\text { C- } \\
\text { D }\end{array}$} & \multirow[b]{2}{*}{ D-E } & \multirow[b]{2}{*}{$>E$} & \multirow[b]{2}{*}{ A-IO } & \multirow[b]{2}{*}{$\begin{array}{l}\text { IO- } \\
\text { LS }\end{array}$} & \multirow[b]{2}{*}{$\begin{array}{l}\text { LS- } \\
\text { CP }\end{array}$} & \multirow[b]{2}{*}{$>\mathrm{CP}$} & \multirow[b]{2}{*}{ Total } \\
\hline Step & $\begin{array}{l}\text { Monitored } \\
\text { Displ }\end{array}$ & Base Force & A-B & & & & & & & & & \\
\hline & $\mathrm{m}$ & $\mathrm{kN}$ & & & & & & & & & & \\
\hline 0 & 0 & 0 & 12340 & 0 & 0 & 0 & 0 & 12340 & 0 & 0 & 0 & 12340 \\
\hline 1 & 0.256232 & 9380.9765 & 12340 & 0 & 0 & 0 & 0 & 12340 & 0 & 0 & 0 & 12340 \\
\hline 2 & 0.512464 & 18761.9022 & 12340 & 0 & 0 & 0 & 0 & 12340 & 0 & 0 & 0 & 12340 \\
\hline 3 & 0.650146 & 23802.5745 & 12339 & 1 & 0 & 0 & 0 & 12340 & 0 & 0 & 0 & 12340 \\
\hline 4 & 0.910199 & 32997.5329 & 12293 & 47 & 0 & 0 & 0 & 12340 & 0 & 0 & 0 & 12340 \\
\hline 5 & 1.117115 & 38629.8446 & 12224 & 116 & 0 & 0 & 0 & 12339 & 1 & 0 & 0 & 12340 \\
\hline 6 & 1.177139 & 39556.0535 & 12197 & 143 & 0 & 0 & 0 & 12334 & 6 & 0 & 0 & 12340 \\
\hline 7 & 1.234714 & 40005.646 & 12187 & 153 & 0 & 0 & 0 & 12329 & 11 & 0 & 0 & 12340 \\
\hline 8 & 1.292865 & 40255.6117 & 12177 & 163 & 0 & 0 & 0 & 12329 & 11 & 0 & 0 & 12340 \\
\hline 9 & 1.30465 & 40279.0073 & 12175 & 165 & 0 & 0 & 0 & 12323 & 17 & 0 & 0 & 12340 \\
\hline 10 & 1.340129 & 40295.8137 & 12171 & 169 & 0 & 0 & 0 & 12310 & 29 & 0 & 1 & 12340 \\
\hline 11 & 1.377395 & 40330.2606 & 12168 & 172 & 0 & 0 & 0 & 12285 & 53 & 0 & 2 & 12340 \\
\hline 12 & 1.409855 & 40344.9873 & 12164 & 176 & 0 & 0 & 0 & 12272 & 66 & 0 & 2 & 12340 \\
\hline 13 & 1.414774 & 40349.9646 & 12164 & 175 & 1 & 0 & 0 & 12270 & 68 & 0 & 2 & 12340 \\
\hline 14 & 1.415434 & 40373.664 & 12164 & 175 & 1 & 0 & 0 & 12270 & 68 & 0 & 2 & 12340 \\
\hline
\end{tabular}

In X direction pushover, it can be seen that almost the entire structure is still able to withstand the force. 


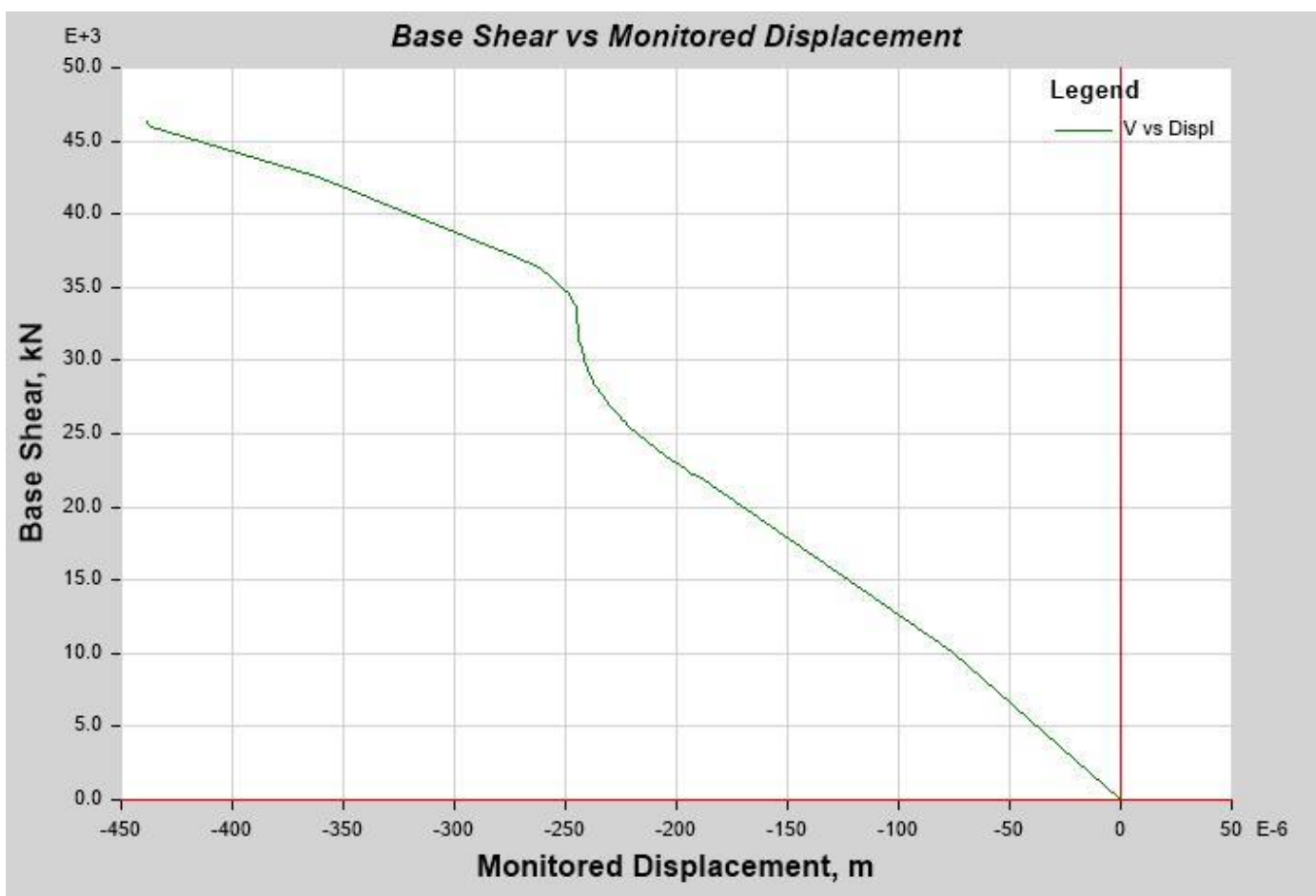

Picture 13. Graphic Displacement $Y$ direction

Table 11 : Result Table Displacement $Y$

TABLE: Base Shear vs Monitored

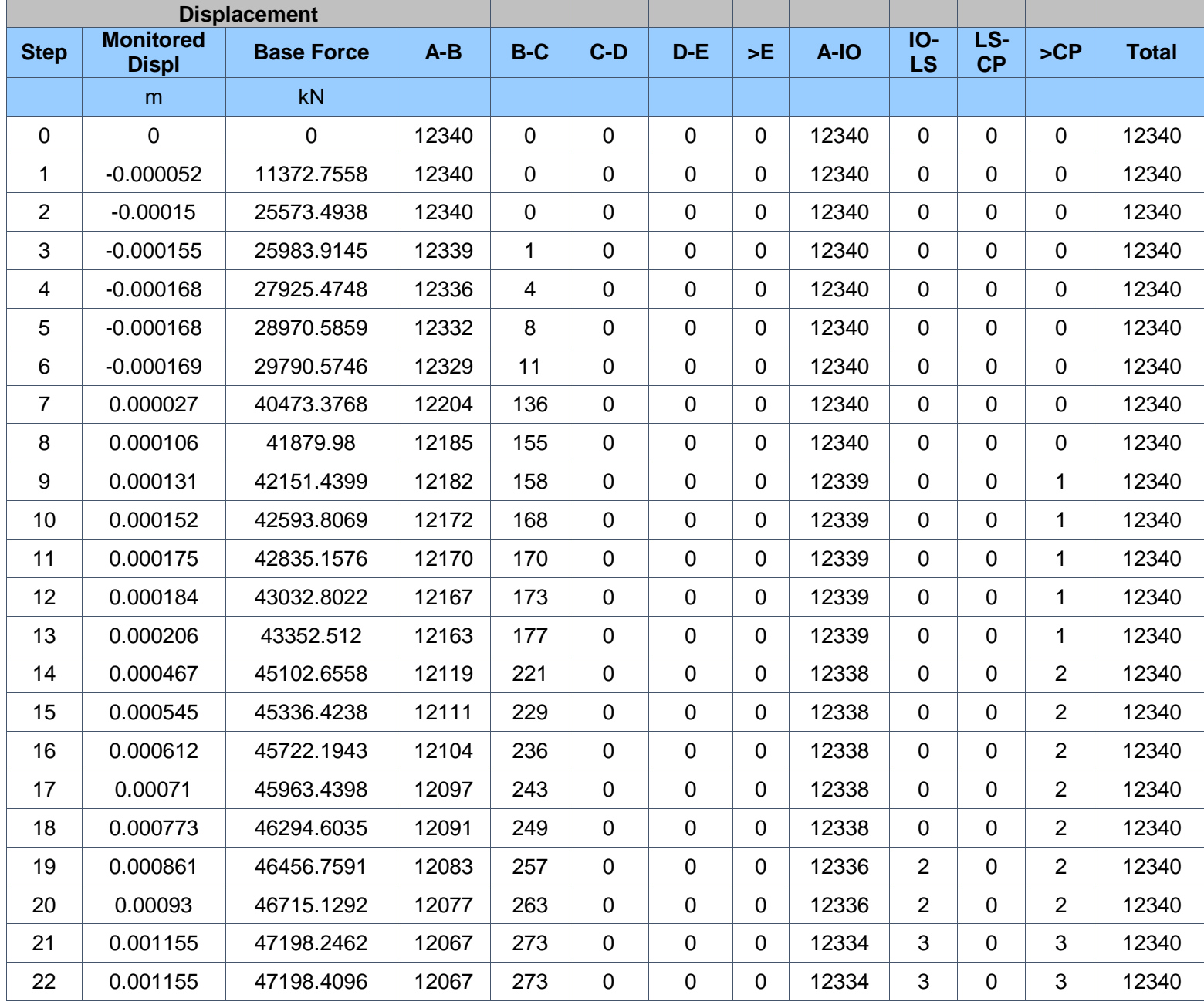




\begin{tabular}{|c|c|c|c|c|c|c|c|c|c|c|c|c|}
\hline \multicolumn{4}{|c|}{$\begin{array}{c}\text { TABLE: Base Shear vs Monitored } \\
\text { Displacement }\end{array}$} & \multirow[b]{2}{*}{ B-C } & \multirow[b]{2}{*}{ C-D } & \multirow[b]{2}{*}{ D-E } & \multirow[b]{2}{*}{$>E$} & \multirow[b]{2}{*}{ A-1O } & \multirow[b]{2}{*}{$\begin{array}{l}\text { IO- } \\
\text { LS }\end{array}$} & \multirow[b]{2}{*}{$\begin{array}{l}\text { LS- } \\
\text { CP }\end{array}$} & \multirow[b]{2}{*}{$>\mathrm{CP}$} & \multirow[b]{2}{*}{ Total } \\
\hline Step & $\begin{array}{l}\text { Monitored } \\
\text { Displ }\end{array}$ & Base Force & A-B & & & & & & & & & \\
\hline & $\mathrm{m}$ & kN & & & & & & & & & & \\
\hline 23 & 0.001155 & 47198.8926 & 12067 & 273 & 0 & 0 & 0 & 12334 & 3 & 0 & 3 & 12340 \\
\hline 24 & 0.001155 & 47199.1705 & 12067 & 273 & 0 & 0 & 0 & 12334 & 3 & 0 & 3 & 12340 \\
\hline 25 & 0.001156 & 47220.1641 & 12067 & 273 & 0 & 0 & 0 & 12334 & 3 & 0 & 3 & 12340 \\
\hline 26 & 0.001156 & 47220.4347 & 12067 & 273 & 0 & 0 & 0 & 12334 & 3 & 0 & 3 & 12340 \\
\hline 27 & 0.001156 & 47220.9267 & 12067 & 273 & 0 & 0 & 0 & 12334 & 3 & 0 & 3 & 12340 \\
\hline 28 & 0.001156 & 47220.9439 & 12067 & 273 & 0 & 0 & 0 & 12334 & 3 & 0 & 3 & 12340 \\
\hline
\end{tabular}

From the table above it can be seen that most structures can withstand loads in the $Y$ direction, but some structures that experience collapse can have a major effect on other structures.

\subsection{Performance Structure}

After obtaining the results from the pushover calculation, the structure performance level is determined based on the ATC-40.

Table 12 : Structure Performance Level ATC-40

\begin{tabular}{|c|c|c|c|c|}
\hline Deviation & \multicolumn{4}{|c|}{ Structure Performance Level } \\
\cline { 2 - 5 } $\begin{array}{c}\text { Limits } \\
\text { Between } \\
\text { Levels }\end{array}$ & $\begin{array}{c}\text { Immediate } \\
\text { Occupancy }\end{array}$ & $\begin{array}{c}\text { Damage } \\
\text { Control }\end{array}$ & $\begin{array}{c}\text { Life } \\
\text { Safety }\end{array}$ & $\begin{array}{c}\text { Strucutral } \\
\text { Stability }\end{array}$ \\
\hline $\begin{array}{c}\text { Maximum } \\
\text { Total } \\
\text { Deviation }\end{array}$ & 0.01 & $\begin{array}{c}0.01- \\
0.02\end{array}$ & 0.02 & $\begin{array}{c}0.33 \mathrm{Vi} / \\
\mathrm{Pi}\end{array}$ \\
\hline $\begin{array}{c}\text { Maximum } \\
\text { Inelastic } \\
\text { Deviation }\end{array}$ & 0.005 & $\begin{array}{c}0.005- \\
0.015\end{array}$ & $\begin{array}{c}\text { No } \\
\text { limits }\end{array}$ & No limits \\
\hline
\end{tabular}

Source : ATC-40,1996 Table 11-2

Table 13

Rasio X Direction

\begin{tabular}{|c|c|c|}
\hline $\mathrm{D}(\mathrm{m})$ & $\mathrm{H}(\mathrm{m})$ & Rasio \\
\hline 1.415 & 128.2 & 0.011037441 \\
\hline
\end{tabular}

Table 14.

Rasio Y Direction

\begin{tabular}{|c|c|c|}
\hline $\mathrm{D}(\mathrm{m})$ & $\mathrm{H}(\mathrm{m})$ & Rasio \\
\hline 0.001 & 128.2 & $7.80031 \mathrm{E}-06$ \\
\hline
\end{tabular}

\section{CONCLUSIONS}

From the table of the ratio of the $X$ direction and $Y$ direction, the level of performance of the structure in the $X$ direction is Damage Control, while for the $Y$ direction it is Immediate Occupancy. With this the performance level of the structure for security has been reached. It is necessary to continue with a more detailed study to obtain an accurate design, so that the design can be like the conditions in the field with suitable results.

In the column, column HC $582 \times 500 \times 50 \times 65$ has a capacity to withstand a force of $18799 \mathrm{kN}$ a moment of $4320.3 \mathrm{kN}$ and a shear force of $3026 \mathrm{kN}$, column HC $508 \times 462 \times 75 \times 75$ has a capacity to withstand a force of $20475 \mathrm{kN}$ a moment of $3885 \mathrm{kN}$ and a shear force of $3595.7 \mathrm{kN}$. In a beam, a WF $400 \times 300 \times 12 \times 22$ beam has a capacity to withstand a force of $3502.8 \mathrm{kN}$ a moment of 
$641.7 \mathrm{kN}$ and a shear force of $572.1 \mathrm{kN}$, a WF $400 \times 200 \times 12 \times 22$ beam has a capacity to withstand a force of $2225.4 \mathrm{kN}$ a moment of $456 \mathrm{kN}$ and a shear force of $572.1 \mathrm{kN}$.

In conclusion, the planned columns and beams are strong but quite wasteful.

\section{REFERENCES}

1. Tavio, Wijaya. 2018. Desain Rekayasa Gempa Berbasis Kinerja (Performance Based Design). Yogyakarta : ANDI.

2. Dewobroto. 2016. Struktur Baja Perilaku, Analisis \& Desain - AISC 2010 Edisi ke 2. Jakarta : Universitas Pelita Harapan, Lumina Press.

3. Salmon, Johnson,Wira. 1994. Struktur Baja, Disain dan Perilaku Jilid 1 Edisi ke 2. Jakarta : Erlangga

4. Potma, De Vries, Hendrardji. 2001. Konstruksi Baja, Teori Perhitungan dan Pelaksanaan. Jakarta : Pradnya Paramita

5. Badan Standarisasi Nasional. 2019. SNI 1726 : 2019. "Tata Cara Perencanaan Ketahanan Gempa Untuk Struktur Bangunan Gedung dan Nongedung". Jakarta. : BSN

6. Badan Standarisasi Nasional. 2013. SNI 1727 : 2013. "Beban Minimum untuk Perancangan Bangunan Gedung dan Struktur Lain". Jakarta : BSN

7. Badan Standarisasi Nasional. 2015. SNI 1729 : 2015. "Spesifikasi untuk Bangunan Gedung Baja Struktural". Jakarta : BSN

8. American Institute of Steel Construction. 2010. ANSI/AISC 360-10. "Specification for Structural Steel Buildings". Chicago : ANSI

9. American Institute of Steel Construction. 2010. ANSI/AISC 341-10. "Seismic Provisions for Structural Steel Buildings". Chicago : ANSI

\section{BIOGRAPHIES}

Tony Hartono Bagio is Lecturer at Narotama University. S1-Bachelor of Civil Engineering - UKPetra, S2- Masters in Informatics Engineering - ITS. Master of Management at Narotama University.

Julistyana Tistogondo is Lecturer at Narotama University. S1- Bachelor of Civil Engineering UKPetra, S2- Master

of Civil Engineering at Universitas Kristen Indonesia.

Achmad Ubaidillah is college student at Civil Engineering Faculty of Narotama University. 